\title{
Acute Myocardial Infarction and COVID-19 in a Known Case of Granulomatosis with Polyangiitis
}

\author{
Seyyed Hossein Mousavi, MD; Seyyed Reza Raeeskarami, MD²; Vahideh Laleh far, MD; Reza Mollazadeh, MD $^{3 *}$ \\ ${ }^{1}$ Department of Cardiology, School of Medicine, AJA University of Medical Sciences, Tehran, Iran \\ ${ }^{2}$ Department of Pediatrics, Imam Khomeini Hospital Complex, Tehran University of Medical Sciences, Tehran, Iran \\ ${ }^{3}$ Department of Cardiology, Imam Khomeini Hospital Complex, Tehran University of Medical Sciences, Tehran, Iran
}

\begin{abstract}
Since the emergence of novel coronavirus and the disease named as COVID-19 in late December of 2019 in Wuhan, Hubei province, China, many aspects of this disease have been reported in the literature (mainly pulmonary manifestations). In patients with COVID-19, rheumatic and cardiovascular manifestations and interactions were reported separately, but they were all very rare. This is the report of a 14-year-old teenager with GPA (previously known as Wegner's granulomatosis) who was in remission with immunosuppressive therapy. Post COVID-19 infection, she developed exacerbation of her disease. Besides the rheumatologic manifestations, she developed epigastric pain found to be acute myocardial infarction (MI) that needed primary percutaneous coronary intervention $(\mathrm{PCl})$.

Keywords: COVID-19, Granulomatosis, Myocardial infarction, Polyangiitis, SARS-CoV-2

Cite this article as: Mousavi SH, Raeeskarami SR, Laleh far V, Mollazadeh R. Acute myocardial infarction and Covid-19 in a known case of granulomatosis with polyangiitis. Arch Iran Med. 2021;24(4):339-340. doi: 10.34172/aim.2021.48
\end{abstract}

Received: November 17, 2020, Accepted: January 31, 2021, ePublished: April 1, 2021

\section{Introduction}

In December 2019, pandemic of pneumonia occurred in Wuhan, China due to severe acute respiratory syndrome coronavirus 2 (SARS-CoV-2). ${ }^{1}$ In addition to respiratory symptoms, there are recent reports of other organ involvements in the course of the disease, e.g. cardiac involvement, ${ }^{2-4}$ rheumatic diseases ${ }^{5}$ and nervous system. ${ }^{6}$

Cardiovascular system and rheumatologic interactions in the context of COVID-19 is very rare. We have previously reported flare-up of hypereosinophic syndrome in the context of COVID-19.5 Herein we present a case of granulomatosis and polyangiitis (GPA) (previously known as Wegner's granulomatosis).

\section{Case Report}

A 14-year-old teenager was referred to our hospital during the COVID-19 outbreak in August 2020 due to new onset epigastric pain since the night prior to admission. She was diagnosed as a case of GPA since 2 months prior to admission based on sinus tissue biopsy following workup for chronic sinusitis and arthritis. Her symptoms were relieved after treatment with high dosage of prednisolone and azathioprine.

During the recent admission, her chief complaints were epigastric pain and skin lesions on the dorsum of her feet (Figure 1A). Vital signs were stable and she was afebrile. Except for skin lesions, physical examination was unremarkable. Chest $\mathrm{X}$ ray was within normal limits. Electrocardiography (ECG) showed ST segment elevation in the inferolateral leads (Figure 1B). Serum high sensitive troponin $\mathrm{T}$ and NT-proBNP levels were $500 \mathrm{ng} / \mathrm{L}$ and $7515 \mathrm{pg} / \mathrm{mL}$, respectively (both above the upper limit). Echocardiography revealed hypokinesia of apical segments. Emergent coronary angiography depicted thrombotic occlusion of the mid part of the left anterior descending (LAD) artery (Figure 1C). Other coronary vessels were free of atherosclerosis. After successful primary percutaneous coronary intervention (PCI) (Figure 1D), ST segment elevation resolved completely. Serum IgG was positive for the new coronavirus. Aspirin and clopidogrel were added to previous anti-inflammatory regimens. She was discharged after 1 week of hospitalization.

\section{Discussion}

Inflammation and its consequences can link COVID-19, cardiac involvement and GPA. It is now clear that after the viremia phase in COVID-19, there is an inflammatory phase (up to cytokine storm) that can be treated with immunosuppressive (Corticosteroids or immunomodulators, etc.) that may reduce the COVID-19 sudden cardiac death ${ }^{7}$ and total mortality. ${ }^{8}$

The diagnosis of GPA may be challenging during COVID-19 pandemic for several reasons. First, clinical presentation of GPA and COVID-19 may overlap. Second, many patients with GPA may use immunosuppressive agents and are afraid to be into close contact with other patients, and third, diagnosis may be delayed because nonurgent tests and visits might have been postponed due to 


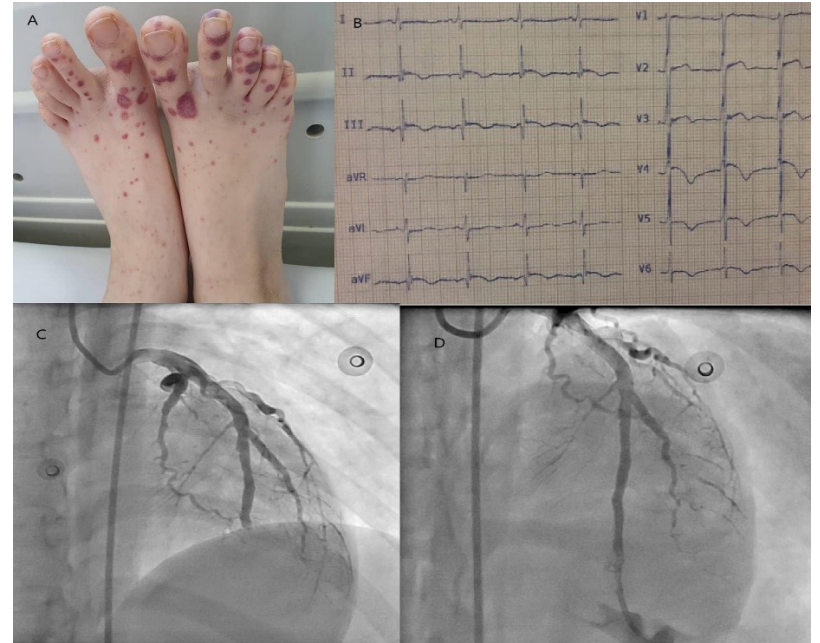

Figure 1. A) Petechiae, purpura and ecchymosis on both feet are seen. B) Electrocardiogram (ECG) shows sinus rhythm, ST-segment elevation and T wave inversion in lead II, III, aVF and V3 through V6. C) Left anterior descending (LAD) is totally cut from mid part. D) Successful percutaneous coronary intervention (PCI) for LAD.

\section{COVID-19 related closure of services. ${ }^{9}$}

On the cardiovascular side, COVID-19 may lead to myocarditis, ${ }^{10}$ bradyarrhythmias and tachyarrhythmia, ${ }^{3}$ myocardial infarction (MI), ${ }^{11}$ as well as worsening of preexisting cardiovascular diseases. Our hypothesis is that increased inflammation caused by host-coronavirus interaction leads to hypercoagulability and nonatherosclerotic MI.

This report emphasizes the challenges of COVID-19, myocardial involvement, and GPA together in one patient. ${ }^{12,13}$

\section{Authors' Contribution}

SHM, SRR and VLF: Images and data. VLF and RM: Manuscript draft. RM: Revision of manuscript.

\section{Conflict of Interest Disclosures}

None.

\section{Ethical Statement}

Informed consent was obtained from the patient for use of photographs and for publication of this case.

\section{References}

1. Guan WJ, Ni ZY, HuY, Liang WH, Ou CQ, He JX, et al. Clinical Characteristics of Coronavirus Disease 2019 in China. N Engl J Med. 2020;382(18):1708-20. doi: 10.1056/NEJMoa2002032.

2. GuoT, Fan Y, Chen M, Wu X, Zhang L, HeT, et al. Cardiovascular Implications of Fatal Outcomes of Patients With Coronavirus Disease 2019 (COVID-19). JAMA Cardiol. 2020;5(7):811-8. doi:10.1001/jamacardio.2020.1017.

3. Coromilas EJ, Kochav S, Goldenthal I, Biviano A, Garan H, Goldbarg S, et al. Worldwide survey of COVID-19 associated arrhythmias. Circ Arrhythm Electrophysiol. 2021. doi: 10.1161/CIRCEP.120.009458.

4. Laleh Far V, Mehrakizadeh A, Eslami M, Shirazi S, Mohammadi M, Mollazadeh R. Electrocardiography Holter monitoring in critically ill patients with coronavirus disease 2019 (COVID-19). Pol Arch Intern Med. 2020;130(11):1010-1012. doi: 10.20452/pamw.15601.

5. Laleh FarV, Najafizadeh SR, Eslami M, Mollazadeh R. A flare up of idiopathic hypereosinophilic syndrome due to COVID-19. Eur Heart J. 2021;42(9):954 doi: 10.1093/eurheartj/ehaa714.

6. Bougakov D, Podell K, Goldberg E. Multiple neuroinvasive pathways in COVID-19. Mol Neurobiol. 2021;58(2):564-575. doi: 10.1007/s12035-020-02152-5.

7. Shirazi S, Mami S, Mohtadi N, Ghaysouri A, Tavan H, Nazari A, et al. Sudden cardiac death in COVID-19 patients, a report of three cases. Future Cardiol. 2021;17(1):113-118. doi: 10.2217/fca-2020-0082.

8. Mishra GP, Mulani J. Corticosteroids for COVID-19: the search for an optimum duration of therapy. Lancet Respir Med. 2021 Jan;9(1):e8. doi: 10.1016/S2213-2600(20)30530-0.

9. Giollo A, Bixio R, Gatti D, Viapiana O, Idolazzi L, Dejaco C, et al. Challenge of diagnosing ANCA-associated vasculitis during COVID-19 pandemic: a missed 'window of opportunity'. Ann Rheum Dis. 2020;annrheumdis-2020-218830. doi: 10.1136/ annrheumdis-2020-218830.

10. Zeng JH, Liu YX, Yuan J, Wang FX, Wu WB, Li JX, et al. First case of COVID-19 complicated with fulminant myocarditis: a case report and insights. Infection. 2020;48(5):773-7. doi: 10.1007/s15010-020-01424-5.

11. Solomon MD, McNulty EJ, Rana JS, Leong TK, Lee C, Sung $\mathrm{S}-\mathrm{H}$, et al. The Covid-19 pandemic and the incidence of acute myocardial infarction. N Engl J Med. 2020;383(7):691-3. doi: 10.1056/NEJMc2015630. Epub 2020 May 19.

12. Duran E, Kilic L, Durhan G, Inkaya AÇ, Guven GS, Karakaya $\mathrm{G}$, et al. Vital corner of diagnostic challenge: eosinophilic granulomatosis with polyangiitis or COVID-19 pneumonia? Ann Rheum Dis. 2020; annrheumdis-2020-218533. doi: 10.1136/annrheumdis-2020-218533.

13. Hakroush S, Franz J, Larsen J, Korsten P, Winkler MS, Tampe B. Repeated false-negative tests delayed diagnosis of COVID-19 in a case with granulomatosis with polyangiitis under maintenance therapy with rituximab and concomitant influenza pneumonia. Ann Rheum Dis. 2020;annrheumdis-2020-218491. doi: 10.1136/annrheumdis-2020-218491. 